\title{
LA AUDITORIA AMBIENTAL Y SU PROCESO EN EL CONTEXTO DE LA AUDITORIA INTEGRAL
}

Responsable: CPC. Rubén Soto Huanca Miembro: CPC Augusto Cahuapaza Morales

\begin{abstract}
RESUMEN
La auditoria de gestión ambiental, por parte de la Contraloria General de la República, ha previsto mitigar los efectos contaminantes y establecer recomendaciones para mejorar el nivel de la población de la ciudad de Moquegua El éxito de la implantación de un sistema de gestión medio-ambiental, dependerá entre otros factores, del establecimiento de políticas y objetivos adecuados y coherentes de que se instrumente una buena comunicación que alcance a todas las esferas de la empresa y estimule la participación todos los trabajadores, de la creación de responsabilidades para cada uno de los miembros implicados y la utilización de la auditoría medi-ambiental como mecanismo de control que asegure a los responsables el cumplimiento de la política y objetivos establecidos y la idoneidad del sistema implantado, por lo que es de suma importancia su proceso en el contexto de la auditoria integral.
\end{abstract}

\section{ABSTRACT}

The audit of environmental administration on the part of the General Controllership of the Republic, if they have foreseen to mitigate the polluting goods and to establish recommendations to improve the level of the population of the city of Moquegua The success of the installation of a system of half environmental administration it will depend among other factors of the establishment of political and appropriate and coherent objectives that I know it orchestrates a good communication that reaches to all the spheres of the company and stimulate the participation of all the workers, of the creation of responsibilities for each one of the implied members, and of the use of the half environmental audit as control mechanism that assures to the responsible ones the politics's execution and established objectives and the suitability of the implanted system, for what is of supreme importance their process in the context of the integral audit.

\section{INTRODUCCIÓN}

La acción del auditor es amplisima, por lo que en este campo podrán hacerse nominaciones de carácter general considerando establecer sus funciones especificas. Así determinar la auditoria de gestión ambiental por parte de la Contraloria General de la República si han previsto mitigar los efectos contaminantes y establecer recomendaciones para mejorar el nivel de la población por efectos de las actividades mineras. El auditor deberá reunir requisitos básicos que encierren una formación de trabajo, experiencia y metodología práctica, iniciando el trabajo con un cuestionario o modelo donde se recabará información general del plan de gestión medio-ambiental aplicado en la empresa; se seguirá con un estudio económico sobre costo de gestión y legislación ambiental. Además el auditor debe tener conocimientos medio-ambientales sólidos, ya que estamos frente a una auditoría muy especial, la implantación de la Auditoria Ambiental dentro del contexto de la Auditoria Integral que tiene un abanico de acción diversificado, atendiendo al tipo de actividad y tamaño empresarial, considerando adecuadamente la legislación medio-ambiental en el ámbito nacional, regional o internacional, según el caso. Con relación a las normas generales de auditoria aceptadas.

"Naga", son plenamente aplicables y los procedimientos técnicos no difieren mayormente, ya que los balances no se diferencian de los estados generales, en razón de que los recursos asignados pueden cuantificarse y clasificarse indistintamente como gastos o inversiones según criterio financiero contable; los activos adquiridos para prevenir la contaminación ambiental tendrán su clasificación acorde con su destino, los pasivos podrian visualizarse como las normas de una estructura preventiva.

La parte compleja de la Auditoria Ambiental dentro del contexto de la Auditoria Integral se presenta cuando debemos valorizar o depreciar la parte relacionada con los recursos naturales debido a la dificultad de evaluación de los activos y/o pasivos. Es aquí donde encontramos que no hay una medición normada; pero sí encontramos, desde el punto de vista ambiental, fundamentos, principios y objetivos de una política ambiental.

El desarrollo sustentable proporciona 
marco para la integración de las políticas ambientales y las estrategias de desarrollo social y económico y reconoce que el crecimiento es esencial para satisfacer las necesidades humanas y para mejorar la calidad de vida; sin embargo, el desarrollo debe basarse en el uso eficiente, equitativo y ambientalmente responsable de todo el recurso escaso de la sociedad, es decir, los recursos naturales, humanos y económicos:

\section{Los Fundamentos de una Política Ambiental.}

- Calidad de vida de las personas

- Complementariedad entre el desarrollo socioeconómico y la sustentabilidad ambiental.

- Equidad social y superación de la pobreza

\section{Principios de Política Ambiental}

- Políticas públicas ambientalmente sustentables.

- Roles de los estados y de los privados

- Prevención.

- Gradualismo y mejoramiento continuo.

- Responsabilidad ante la comunidad internacional.

Los Objetivos de una Politica Ambiental los podemos resumir:

Promover la sustentabilidad ambiental del proceso de desarrollo con miras a mejorar la calidad de vida de los ciudadanos, garantizando un medio ambiente libre de contaminación. La protección del medio ambiente, la preservación de la naturaleza y la conservación del patrimonio ambiental, perfeccionando la legislación ambiental y desarrollar instrumentos de gestión.

La auditoria de gestión ambiental por parte de la Contraloría General de la República si han previsto mitigar los efectos contaminantes y establecer recomendaciones para mejorar el nivel de la población.

\section{MARCOTEÓRICO}

Como antecedente la auditoría de gestión ambiental es un medio para determinar los efectos contaminantes de las empresas basado en la auditoría de gestión y en la auditoría de cumplimiento o desempeño como un examen sistemático, profesional, independiente y objetivo sobre las políticas, planes objetivos y metas, cumplimiento de la normativa aplicable a programas, proyectos de inversión y demás actividades de las entidades, tendentes a prevenir y/o mitigar los daños ambientales. Tiene la finalidad de coadyuvar con la gestión de las entidades a favor de la conservación del medio ambiente y del uso racional de los recursos naturales y presentar propuestas de concientización ambiental de autoridades y funcionarios públicos y privados, asi como de la población, a fin de contribuir con el desarrollo sostenible del país.

Para verificar el cumplimiento de las normas ambientales del sector minero, el Ministerio cuenta con el Decreto Ley 25763 del 11 de octubre de 1992 para efectuar la fiscalización a través de terceros. El Decreto Supremo 012-93-EM, del 4 de marzo de 1993, "Reglamento de Fiscalización de las Actividades Minero Energéticas por terceros", establece las normas reglamentarias que permiten el ordenamiento del proceso de fiscalización.

Consejo Nacional del Ambiente (CONAM) fue creado por Ley $N^{\circ} 26410$ del 2 de diciembre de 1994. Es el organismo rector de la politica ambiental y tiene entre sus funciones:

1. El establecer los criterios y patrones de calidad ambiental.

2. La coordinación referente a la fijación de los limites permisibles para la protección ambiental.

\section{El establecimiento de los criterios.}

En 1996, la Universidad de Kent realizó un estudio para establecer si las intenciones y actitudes de los empresarios hacia la protección ambiental eran consecuentes con las acciones reales. El resultado del estudio: la ley era el verdadero motor para el mejoramiento ambiental, aunque todos consideraban que el aspecto ambiental es fundamental (imagen y costos) para hacer empresa.

Luego de dos años, se puede observar un cambio en esta situación, ya que la actitud empresarial se dirige a llevar a cabo acciones voluntarias, más allá de los que exige la ley. Los plazos de adecuación ambiental son relativamente cortos si comparamos con otros paises. Para los EIA el cumplimento es inmediato, para los PAMA es 5 y 10 años, dependiendo de la fase de operación.

En ese sentido, para las operaciones antiguas, o existentes, esta adecuación es un costo en el corto plazo, por los cambios que se deben realizar. Sin embargo, a largo plazo puede ser económico.

Para las operaciones nuevas el impacto económico es menor porque se incluye el aspecto ambiental, siendo parte de la inversión.

Lo fundamental es que la minería viene demostrando estar comprometida en operar de manera económica, eficiente, responsablemente en cuestiones ambientales y sociales. 
Entrando ya al tema que se me ha solicitado abordar, quisiera aprovechar la oportunidad para tratar un asunto ambiental de particular importancia, el cual se refiere a la autoridad competente en materia ambiental.

Al respecto, considero que los peruanos en general debemos comprender y aceptar que la fiscalización y el control de los asuntos ambientales mineros le corresponde, según Ley, única y estrictamente a la Autoridad Sectorial. Para el caso de la minería, energía y petróleo, dicha autoridad es el Ministerio de Energía y Minas, que está representada por la Dirección General de Asuntos Ambientales (DGAA), que actúa en coordinación del CONAM.

Por tanto, todas las regulaciones, acciones y sanciones son competencia de la Autoridad Sectorial competente, y no de otros organismos estatales o autoridades ajenas a la sectorial, salvo, claro está, en los casos de delitos contra el medio ambiente en donde será competente el Poder Judicial.

\section{METOdOLOGÍA}

Esta investigación fue de tipo descriptivo, se han empleado técnicas de análisis documental e interpretación de datos, previa recolección de estos a través de un cuestionario, encuestas, entrevistas y revisión de bibliografia y documental, por lo que permite la adopción de medidas en cuanto a la protección del medio ambiente a través de la auditoría ambiental dentro del contexto de la auditoría integral. La auditoría de gestión ambiental por parte de la Contraloria General de la República y otras entidades si han previsto mitigar los efectos contaminantes y establecer recomendaciones para mejorar el nivel de la población afectada.

\section{RESULTADOSYANÁLISIS}

Resultados obtenidos referentes a la auditoria ambiental y su proceso en el contexto de la auditoria Integral es un proceso objetivo independiente de evaluación, orientado al desarrollo organizacional en un entorno de cambio permanente, tal es el caso de la contaminación, cuya gestión puede ser llevada a cabo desde la organización misma en cuanto al valor agregado que aportan los procesos y a la minimización de los riesgos en la organización.

Las auditorias ambientales por parte de la Contraloría General de la Repúbiica no son eficaces y no tienen un control sobre las empresas respecto a las medidas tendientes a mitigar los efectos contaminantes ni establecer recomendaciones para mejorar el nivel de la población, tal como establece el Código de Medio Ambiente.

Es la auditoría ambiental un nuevo esquema perceptivo que permite ver las oportunidades que ofrece la realidad en función de la contaminación. Un modelo epistemológico que nos ayuda a desarrollar las demandas globales del entorno. La auditoria ambiental y su proceso en el contexto de la auditoria integral nos permite la aplicación de un sistema de gestión medio ambiental que dependerá, entre otros factores, del establecimiento de políticas y objetivos adecuados y coherentes de que se instrumente una buena comunicación que alcance a todas las esferas de la empresa y estimule la participación de todos los trabajadores, de la creación de responsabilidades para cada uno de los miembros implicados, de la adopción de un programa adecuado de formación del personal que garantice su capacitación en estos asuntos y de la utilización de la auditoría medioambiental como mecanismo de control que asegure a los responsables el cumplimiento de las politicas y objetivos establecidos y la idoneidad del sistema implantado.

La auditoria ambiental y su proceso en el contexto de la auditoría integral no se aplica por parte del Gobierno Central ni mucho menos por parte de las empresas, por lo que la contaminación está llegando a extremos en el mundo y en el Perú.Las auditorias ambientales por parte de la Contraloría General de la República no son eficaces y no tienen un control sobre la mayoria de empresas que permitan mejorar la calidad de vida de la población respecto a las medidas y los efectos contaminantes ni establecer recomendaciones para mejorar el nivel de vida de acuerdo con la normatividad de protección de la vida como fin supremo del Estado.

El Estado, por medio de la Contraloría General de la República, a través del Ministerio de Energía y Minas, puede auditar a las empresas a fin de cautelar la correcta administración de los recursos para la conservación del medio ambiente y el cumplimiento de la legislación pertinente.

Las empresas no cumplen adecuadamente con las técnicas y procedimientos de auditoria ambiental ni el programa de adecuación y manejo ambiental tal como lo establece el Estado Peruano, porque carece del programa de acciones políticas e inversiones destinadas a la adecuación gradual a nuevas exigencias ambientales, a través de la incorporación de prácticas de prevención de la contaminación, implementación de tecnologías, cambios en los procesos de producción, operación y usos de insumos con el objeto de reducir prioritariamente la contaminación ambiental. 


\section{CONCLUSIONES}

1. La auditoria ambiental y su proceso en el contexto de la auditoria integral no se aplica por parte del Gobierno Central ni mucho menos por parte de la empresa, por lo que la contaminación está llegando a extremos en el mundo y en el Perú, caso ciudad de Moquegua.

2. Las auditorias ambientales por parte de la Contraloria General de la República no son eficaces y no tienen un control sobre las empresas y sus efectos contaminantes; ni establecen recomendaciones para mejorar el nivel de vida de la población.

3. El Estado, por medio de la Contraloria General de la República, a través del Ministerio de Energía y Minas, puede auditar a las empresas a fin de cautelar la correcta administración de los recursos para la conservación del medio ambiente y el cumplimiento de la legislación pertinente.

4. Las Empresas no cumplen adecuadamente con las técnicas y procedimientos de aucitoría ambiental ni el programa para el cumplimiento del Programa de Adecuación y Manejo Ambiental (PAMA).

5. La contaminación ambiental esta llegando a tales extremos en el mundo y en el Perú, que el ser humano parece estar empeñado en destruir el ambiente donde vive, en una actitud degradante; pero mientras que en otros paises se están tomando medidas muy serias para prevenir y controlar la contaminación, en el nuestro solo existe acciones aisladas .

6. El problema ambiental afecta al bien común y a la calidad de vida $y$, en consecuencia, no puede quedar al libre albedrio de las personas y/o empresas; el bien común es una responsabilidad del Estado como representante del bienestar de todos los ciudadanos; esa responsabilidad genera derechos y obligaciones del Gobierno
Central, los gobiernos locales y gobiernos regionales tales como el problema de la contaminación de las empresas industriales, empresas mineras, de las aguas servidas, del control del parque automotor, de las áreas verdes, el control de los ruidos molestos, del ornato y de las emisiones contaminantes en su jurisdicción; pero englobados dentro del contexto de la auditoria ambiental y su proceso en el contexto de la auditoria integral.

7. Los ciudadanos debemos tomar conciencia del problema y cautelarlo y no permitir que las empresas sean indiferentes a los daños irreparables que vienen ocasionando; exigiendo respeto al medio ambiente y no contribuir a su deterioro. Dicho aporte es importante en la educación a la población a través de las instituciones educativas, universidades, medios de comunicación( televisión, radio, periódicos, Internet, etc.) indicando expresamente el conocimiento de la educación ambiental que permita erradicar las pésimas costumbres de contaminación ambiental ai corto, mediano y largo plazo, especialmente de aquellos que tienen mayor impacto sobre la salud y los medios de vida.

\section{BIBLIOGRAFIA}

Vargas, Carlos. Ecologia General. Edito. Taller Gráficos Courier S.A. 1997. Lima Perú.

II Simposium Internacional de Educación en Ciencias Empresariales. 2000, Cusco-Perú.

Arthur W, Colmes. Principios básicos de auditoría. S.A.

Tejada R. Alejandro. Auditoría. Editorial Imnreso.

Auditoria en el Siglo XXI, 1999, Cusco.

Código del Medio Ambiente, 2002, Lima.

Bis Balbin, Doris. Agua Minera y Contaminación en el caso de Souther Peru Copper Corporation.

Documentos, Guías e Impresos Varios de Auditoria Ambiental y Gestión. 